\title{
ACOUSTIC SIGNAL CONTROL IN DISC VORTEX CHAMBER OF HYDRODYNAMIC CAVITATOR
}

\author{
Evgueniy Ivanov \\ Nizhny Novgorod State Agricultural Academy, Russia \\ ivanov.e.g@mail.ru
}

\begin{abstract}
The use of acoustic cavitation processes permits to discover new ways to impact on product, to give unusual properties to liquids and their components, to create new technologies. In the vortex cavitators, which are used in this process, the acoustic field is created with a liquid whistle which looks like a disc vortex chamber. Though its design seems to be simple the liquid flows have a complicated structure. Some of these streams create the required acoustic field due to the competing interactions of two streams intersecting at an acute angle: the incoming stream and the peripheral stream. Peripheral flow is the earlier part of the incoming flow, which has completed almost a full revolution along the envelope. Another part of the flows interferes with the correct interaction and consumes energy. Moreover, when the flow from the tangential inlet pipe enters the cylindrical part of the vortex chamber, the effect the coming into corner takes place. Two conjugated toroidal vortices are formed along the shell. The parameters of the peripheral flow within the cycle are different; therefore, the nature of its interaction with the inlet flow is determined by the current phase of the cycle in the interaction zone. By adjusting the angle of ascent of the vortex components, their intensity, phase at the point of convergence of useful input and peripheral flows, it is possible to change the conditions of their interaction, the quality of the generated acoustic signal. The process of formation and development of vortex torus flows is shown, their intensity, both on a simplified analogue and on a disk-shaped vortex chamber. The places of interaction of the torus-shaped flow with the inner surface of the shell are determined, factors influencing the phase of the flow when it meets the inlet flow. The adequacy is proved by numerical methods based on solving the Navier-Stokes equations in the Flow vision software package.
\end{abstract}

Keywords: vortex chamber, flow liquid, turning, vortex pair, mechanism.

\section{Introduction}

The comprehension of nanoworld and the creation of advanced technologic processes basing on its results is a great resource in human activity. If there are local deformations of liquid media with extra high-pressure and its gradients, the traditionally known capabilities (the blending of inclusions, intimate mixing, the providing of homogeneity of properties by volume), as well as new physical effects (highefficient heat, the change in water properties, providing with biological activity) take place. The capability of water for desalination and mineralization etc. is discovered. The creation of short time extra-high pressure points is possible if there is a periodic cavitation at the cost of sequencing of elastic waves through a liquid (for example, water) massif, if $p \geq 3$ bar. In the vacuum phase of waves, the liquid in nuclei is broken and then the caverns are created. In the manometric phase each of these caverns is collapsed at a speed of opposite traffic of the walls which is higher than double sound speed. A very small final volume caused by the collapse provokes an extremely high power density which causes a mutual transition of forms of energy. Besides, the appearance of its unknown forms is also possible. In such a pulse-signal system, if the vacuum phase is relatively long, the accumulation of elastic energy takes place. In the manometric phase there is its concentrating in space and time. It is feasible that these processes would run synchronically in different collapses and constant periodicity. Thus, the influence of secondary waves appeared, as a result of incoherent collapses adjacent caverns will be reduced.

The initial energy is pumped through wave fronts from the sources of sound formation either from magnetostriction or piezoelectric (rod) radiators or from liquid whistles. Liquid whistles, such as Ranque-Hilsch [1] vortex tube, have the advantage of rod radiators, because if they are used, it is possible to create acoustic fields of multikilowatt power. However, they irradiate elastic vibrations on different frequency. In this case:

- in low frequency of acoustic field in liquid, if the vacuum phase is long, each cavern grows up to a considerable size. That is why pulsation and not collapse is created in the collapse phase.

- in prohibitively high frequency the cavern in each cycle cannot grow up to the optimal size and that is why it preserves an insufficient quantity of initial elastic energy. In collapse a necessary energetic level cannot be achieved [2].

This disadvantage is caused by an irrational flow structure in vortex chambers. 
The process of sound generation in the vortex chamber occurs due to the interaction of flows intersecting at an acute angle: the inlet flow from the discharge pipe and the vortex annular flow of a toroidal shape. Moreover, the peripheral circumferential flow is formed by two conjugate toroidal helical flows. The structure of the circumferential peripheral flow along trajectories path changes periodically. At the moment of its meeting with a potential incoming stream, various options for interaction are possible. This can generate a lot of unproductive noise. However, one of the interaction options is the best.

Purpose: control of the process of interaction of a vortex peripheral torus-shaped flow with a potential input flow.

To achieve this goal, it is necessary to solve the following tasks:

1. to reveal the mechanism of formation of a vortex circular torus-shaped flow;

2. establish the structure of the circular toroidal vortex flow;

3. determine the intensity of the components of this circular vortex flow;

4. form the factors that determine the interaction process.

\section{Materials and methods}

When a liquid flow enters the vortex chamber after passing the outlet pipe, it becomes confined by clear opening only by three sides with plane longitudinal walls (Fig.1).

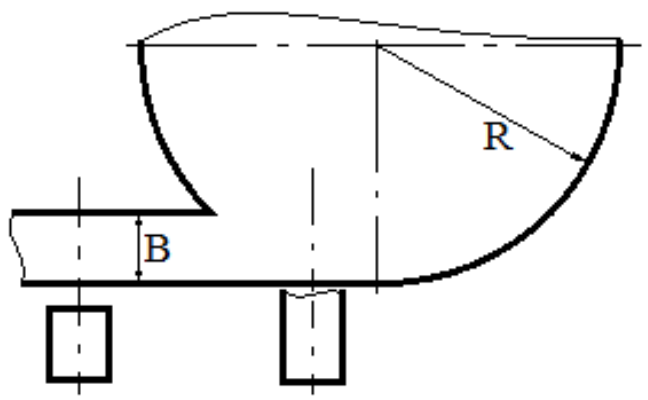

Fig. 1. Schema of change in the flow part in the vortex chamber input

When such flow impinges an obstacle, for example, an angled plane wall (it is a particular case of cylindric wall $R=\infty$ ), which represents a continuation of canal invert different flow components and behaves in different ways. The bottom layers are decelerated earlier when they meet an obstacle and at the same time the capping moves, too (Fig. 2).

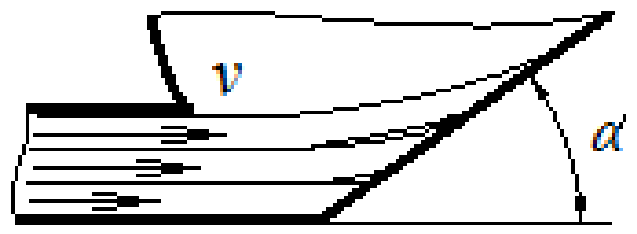

Fig. 2. Trajectory behavior of layer moving in the canal in the impingement on oblique plane obstacle

The kinetic form of energy of low layers transforms to piezometrical and the low layers begin to displace high layers in perpendicular to the speed vector direction. At that, while more remote from the canal invert layers break down, the residuary thickness of the moving flow decreases and it turns aside; in a greater degree by a parabolic curve [3]. This fact was discovered by the author earlier by the solution of the Euler equation system This process takes place till the moving component touches the wall (Fig.3).

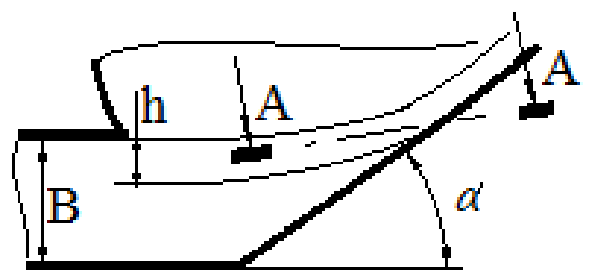

Fig. 3. Structure of initial phase of conjugate toroidal vortexes 
As a result, the initially homogenous flow transforms to a restrained part and to a second component, which moves along the parabola which touches the plane of the obstacle. The tangential path of such flow is caused by the fact that it represents a line with the least hydraulic resistance. It means that itis the most energetically advantageous.

However, this picture of flows is observed in the initial phase of the parabolic flow. In any moment the integrity of the slot parabolic jet destroys in the places of the lowest liquid energy to be exact in wall-adjacent zones - in the frictional boundary layer of lateral parts of the parabolic jet (Fig. 4).

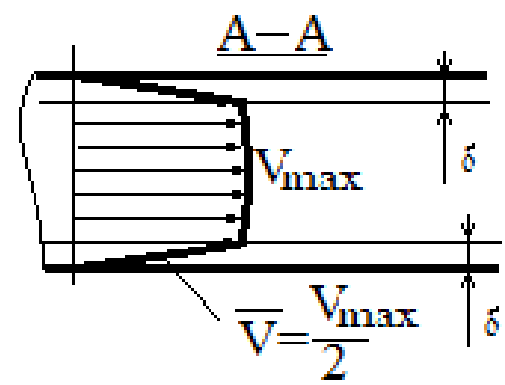

Fig. 4. Structure of flow in parabolic jet (cross-section A-A of Fig. 3)

The flow in frictional boundary layer $\delta$ has less speed and consequently less energy. That is why it is replaced by the low part of the flow stronger than the main parabolic flow. As a result, the integrity is destroyed and two niches are formed in wall-adjacent areas through which the volumes of near-bottom component pass.

The flow velocity is defined by the difference between the possible static pressure in the nearbottom area and the wall-adjacent layer

$$
\Delta p=\rho \frac{V_{1}^{2}}{2}-\rho \frac{\left(0,5 V_{1}\right)^{2}}{2}=\frac{3}{8} \rho V_{1}^{2},
$$

where $\rho$-liquid density, $\mathrm{kg} \cdot \mathrm{m}^{-3}$;

$V_{1}$ - input flow speed, $\mathrm{m} \cdot \mathrm{s}^{-1}$.

It will provide the speed of crossflow $\mathrm{V}_{2}$ from the near-bottom volume through high component

$$
V_{2}=\frac{\sqrt{3}}{2} V_{1},
$$

The replaced layer represents a slot jet [3] twisted by one-sided pressure $\Delta p$ by the parabola

$$
y=\frac{\Delta p}{4 V_{1}^{2} h \rho} x^{2},
$$

where $h$-thickness of the slot jet, $\mathrm{m}$;

$x$ and $y$-coordinates of each flux line of the slot jet, $\mathrm{m}$.

At that the overpressure $\Delta p$ provoking the twist of the slot jet in thickness h (Fig.3) changes from its value in the initial moment corresponding to the complete transition of kinetic energy to the piezometrical

$$
\Delta p=\rho \frac{V_{1}^{2}}{2},
$$

to a smaller value corresponding to the decrease in the pressure caused by the flowing from the nearbottom areas

$$
\Delta p=\mu \rho V_{1}^{2},
$$

where $0.125 \leq \mu \leq 0.5$.

Taking into account the equation (5) the equation (3) will be as follows:

$$
y=\frac{\mu}{4 h} x^{2} .
$$

It is possible to establish the energy balance between the high parabolic and two wall-adjacent centripetal flows by detecting the parabolic pressure rate by determining its thickness $h$. The thickness of the parabolic flow h may be determined due to the following criteria: 
- its touching the oblique line at an obstacle angle $\alpha$;

- overpressure $\Delta p$ determining the degree of parabola twist (6);

- height of the input flow $B$ (Fig. 5).

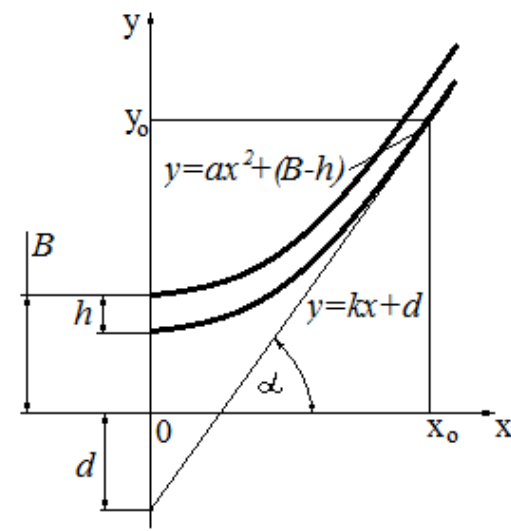

Fig 5. Design diagram in the conditions of touching of the parabolic flow the oblique line at a wall angle $\alpha$

The condition of parabola and tangent common point

$$
a x^{2}+(B-h)=k x+d,
$$

and the parabola differential coefficient, which is equal to the rate of change of the tangent from the touching point $\left(x_{0}, y_{0}\right)$

$$
k=2 a x_{0}
$$

drive to radical

$$
x_{0}=\sqrt{\frac{B-h-d}{a}},
$$

As well as the second coordinate of the touching point

$$
y_{0}=2(B-h)-d \text {. }
$$

The rearrangement of the equation from the standard form to the concrete form, if $x_{0}$ and $y_{0}$, are established:

$$
y=\left(2 a \sqrt{\frac{B-h-d}{a}}\right) x+d,
$$

And the extraction of the coefficient in argument $x$, as well as equation of the coefficient $\alpha$ and the plugging in from (6) drives to

$$
a=\sigma / h,
$$

where $\sigma=\frac{\mu}{4}$.

After the rearrangement

$$
h=\frac{4 \sigma(B-d)}{4 \sigma+(\tan \alpha)^{2}},
$$

or

$$
d=B-h \frac{(\tan \alpha)^{2}+4 \sigma}{4 \sigma} .
$$

Another condition of association of unknown $h, d$ and known $x_{0}, y_{0}$ is the expressing of $d$ as difference

$$
d=x_{0} \tan \alpha-y_{0} .
$$


To be exact

$$
d=\sqrt{\frac{h(B-h-d)}{\sigma}} * \tan \alpha-2(B-h)+d,
$$

inserting (14) drives to

$$
h_{1,2}=\frac{4 B \sigma}{4 \sigma \pm(\tan \alpha)^{2}} .
$$

As a result, it is possible to determine the parabolic flow rate

$$
Q_{\text {par }}=V_{1} * h * H \text {, }
$$

where $H-$ vortex chamber thickness, $\mathrm{m}$.

As well as the rate of each wall-adjacent flows

$$
Q_{1,2}=0,5 * V_{1} *(B-h) * H .
$$

2. The difference between a real concave cylindric obstacle and the mentioned above its simplified analogue which represents a planeangled wall lies in the fact that there is no right complete conjugacy between the parabolic trajectory of jet and plane concave space of the housing (Fig.6).

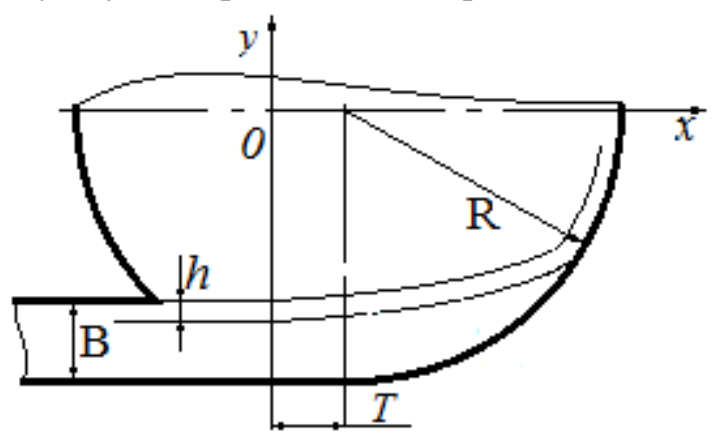

Fig. 6. Scheme of input flow parabolic fragment impingement on concave cylindric obstacle

However, in the sector where the circle and the parabola may cross there is a rendezvous point where the angle between their tangents is minimal. It means that the parabolic flow considered above passing through this point has the minimal loss when it turns, and its trajectories are the lines of least resistance. Consequently, it is possible to suppose that the parabolic flow trajectory will pass through this point and then it will turn along the concave shell of the housing.

The location of this point may be determined by solving an equation obtained by the optimization of the difference between the angles between the parabola tangents $\alpha_{p}$ and circle tangents $\alpha_{c}$ for each of these rendezvous points with coordinates (Fig.7)

$$
x=T+R \cos \varphi, y=R \sin \varphi .
$$

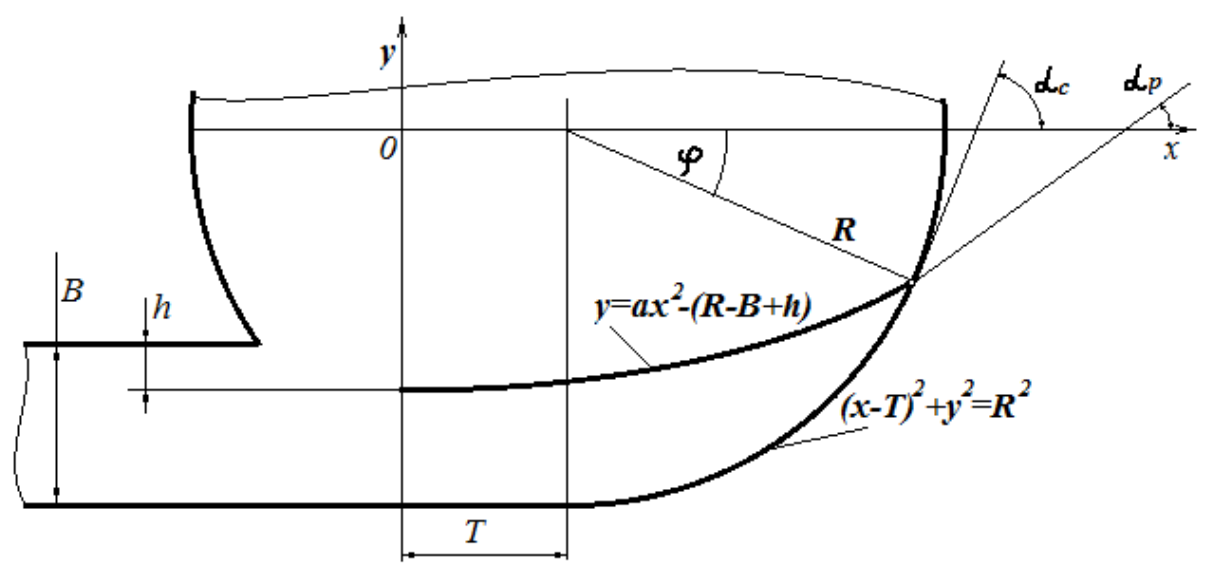

Fig. 7. Design diagram to determinate the parabolic fragment least turn 
At that:

- differential coefficients

- for parabola

$$
y^{\prime}=2 a x,
$$

- for circle

$$
y^{\prime}=\frac{T-x}{\sqrt{R^{2}-(x-T)^{2}}}
$$

- rate of change

- for parabola

$$
k=2 a(T+R \cos \varphi),
$$

- for circle

$$
k=\frac{T-x_{o}}{\sqrt{R^{2}-\left(x_{0}-T\right)^{2}}}=\frac{T-T-R \cos \varphi}{\sqrt{R^{2}-((T+R \cos \varphi)-T)^{2}}}=-\cot \varphi ;
$$

- optimizable function

$$
\Delta \alpha=\tan ^{-1}(2 a(T+R \cos \varphi))-\tan ^{-1}(-\cot \varphi) ;
$$

- extremum equation

$$
1+4 a^{2}(T+R \cos \varphi)^{2}=2 a R \sin \varphi .
$$

According to which $\varphi$ is determined and then the coordinates of point $\left(x_{0}, y_{0}\right)$, through which the least resistance trajectory for the parabolic component of the flow passes is determined by the expressions (20).

The target values $h$ and $T$ are determined by solving the simultaneous equations

$$
\left\{\begin{array}{c}
y_{0}=\frac{\sigma}{h} x_{0}^{2}-(R-B+h) \\
y_{i}=\frac{\sigma}{h} x_{i}^{2}-(R-B)
\end{array},\right.
$$

where $x_{i}, y_{i}$-coordinates of the rendezvous point of the circle and parabola moved in $0 Y$ direction by $h$ quantity.

\section{Results and discussion}

The study of the flow structure in a vortex tube was not made earlier. But earlier the macro flows basically in a cylindrical housing [4-6], and only in aerial working environment were studied. The studies of the flow structure in vortex chambers are also known $[7 ; 8]$, but they touch a unit extended in the rotation axis direction. The competitive flow interaction area is not enough accented. The above discussed process has in some degree a remote analogue. Those are the flows in tube turns [9-11]. But their studies touch circular tubes. Besides, the turn of the tube along all the wetted perimeter of the cross section is boarded with a wall. But in a vortex chambers, only the initial tube entry is completely boarded along the cross section. After the turn the flow flows in open passage. That is to say it is boarded from three sides and the flow deflection is fulfilled by $360^{\circ}$.

The obtained results permit to analyze the flow of two conjugate toroidal vortexes by masse pressure indices, to detect factors influencing the development of this flow, the place of their rendezvous (interaction) with the flow input, the phase of this flow taking part in the interaction. It is feasible to make the analyzes by a more laconic (in comparison with 27) expression (17). The high parabolic flow is considered and it is presumed that the wall-adjacent flows go to the drain system.

The influence of the obstacle slope

- If the obstacle is absent $(\alpha=0)$, the formal parabolic layer thickness is equal to the flow thickness: $h=B$ (Fig. 8). 


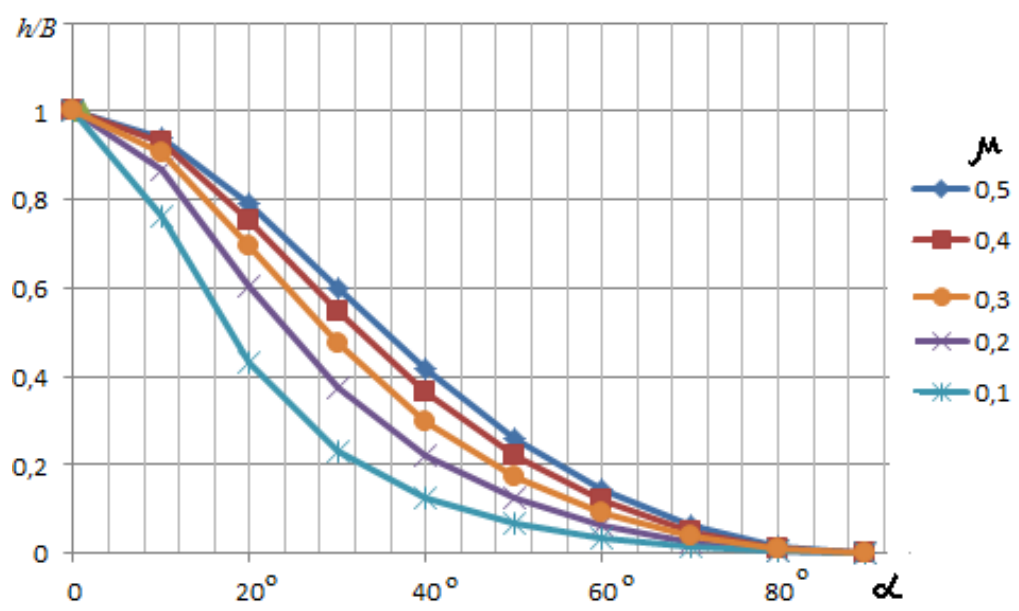

Fig. 8. Result of calculation of the change in $h / B$ depending on the obstacle slope (for different modes $\mu$ )

- If the flow slope in the near-bottom area is small, the zone of statistic pressure is created (4). A small turn provides a sufficient pressure to impact on a bigger thickness of the parabolic component.

- A bigger deformation of the flow parabolic part according to expression (6) presumes its less thickness value $h<B$.

- A considerable deformation of the flow with a steeply established obstacle $\alpha>60^{\circ}$ causes the approximation of the obstacle interfacing surface to the beginning of the turn. As consequence of the above mentioned reason basing on the condition of minimum of the energy expenditure for the tangential flow, it is necessary to twist its trajectory. That is to say to increase the index value in $x^{2}(6)$. The latest condition may be fulfilled at the cost of the decrease in the thickness of the flow parabolic part $h$.

- The most considerable section of $h$ change is the interval of the increase in the obstacle steepness $10^{\circ}<h<70^{\circ}$.

The influence of the correlation between piezometrical and kinetic components of the flow energy $\mu$

The decrease in $\mu$ corresponds to the decrease in the piezometrical and to the increase in the kinetic component of the flow, that is to say, to the process of well adjacent flow intensification. In this case the flow passage of the "pockets" increases. It provokes a grand decrease in the pressure in the nearbottom space.

That is why to provide a required slope of the covering (parabolic) flow fragment due to the condition of its junction with the obstacle surface its thickness has to reduce (Fig. 9).

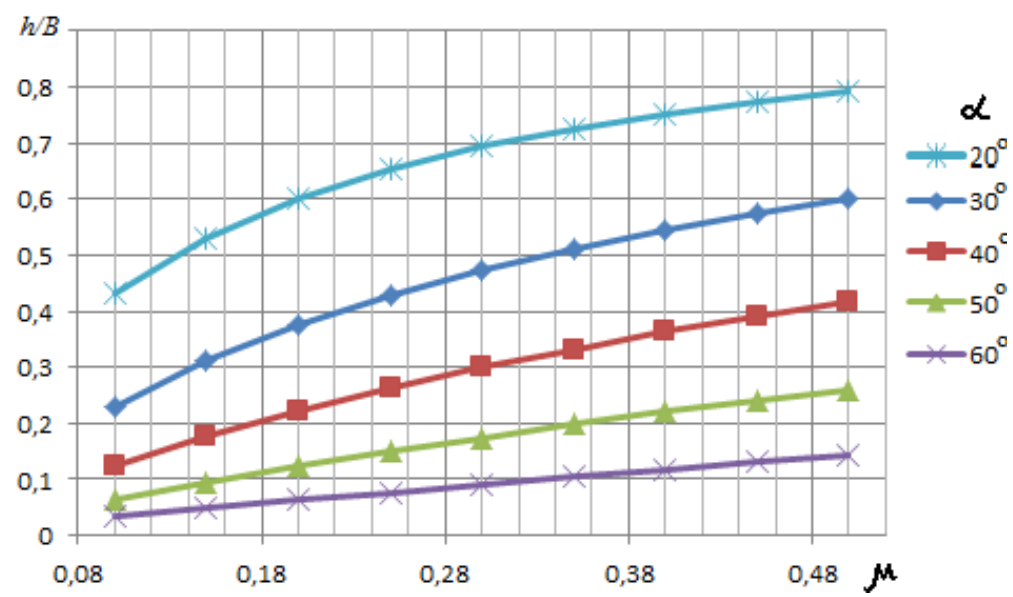

Fig. 9. Calculation results of change in $\boldsymbol{h} / \boldsymbol{B}$ вdepending on correlation between piezometic and kinetic components of the flow energy $\mu$, (for different obstacle slope value $\alpha$ ) 
Meanwhile, the decrease in $\mu$ provokes the enforcement of inequality in static pressure edgeways the canal: it decreases near the walls, but in the middle part it increases square-law of the wall-adjacent flow speed (Fig.10) (dead zone streamlined with sidewinds).

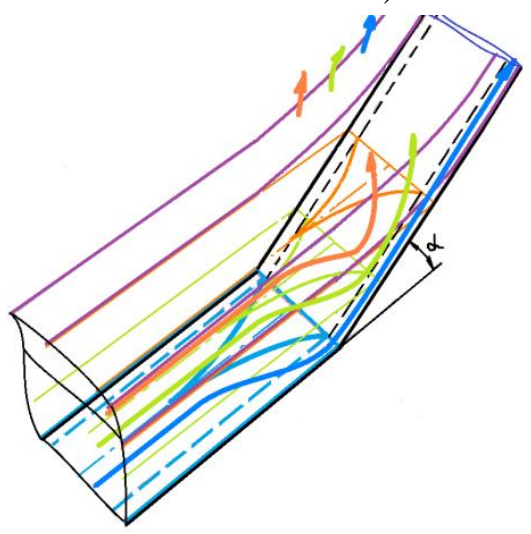

Fig. 10. Structure of flows in "pockets" and the configuration of the dead zone

Then two dispersed in space parallel flows conjugate and the pair of toroidal vortexes appear because of the ejection from the intermediate zone and of the crossing speed appearance.

\section{The influence of the vortex chamber design}

- If the used inlet is narrow, the input flow hits the shell of the vortex chamber in a low-angle slope. Consequently, the thickness of the parabolic part of the flow $h$ will tend to $h$. But as $B$ is small and it is nearly identic with $h$, the toroidal vortexes will be quite small. At that a small angle of impact will predetermine a small reflection angle of the flow active part. The hydraulic losses will increase. Consequently the number of reflection falls will increase (proportionally to the reflection number). But the flow is low power itself. As the result, the influence of the active fragment of peripheral flow on the input flow which is also low rate will be unproductive. In this case the interaction energetics may inconsiderably increase at the cost of the thickness of the vortex chamber itself.

- If the used inlet is wide, the input flow hits the shell of the vortex chamber in a higher angle slope. Consequently it will provoke the decrease in $h$, the increase in the toroidal components, to the displacement of interaction places in the shell. In addition, the interaction zone of input flow and active part of peripheral flow will be nearer to the vortex chamber housing tongue (Fig.11).

a)

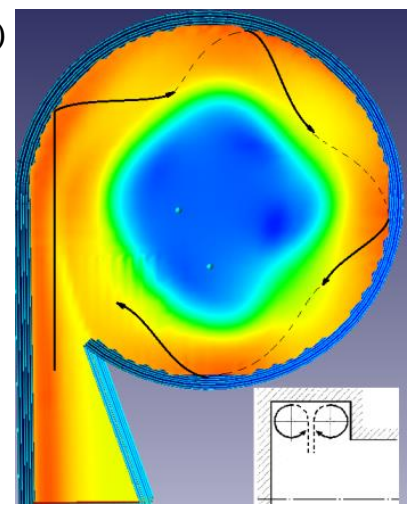

b)

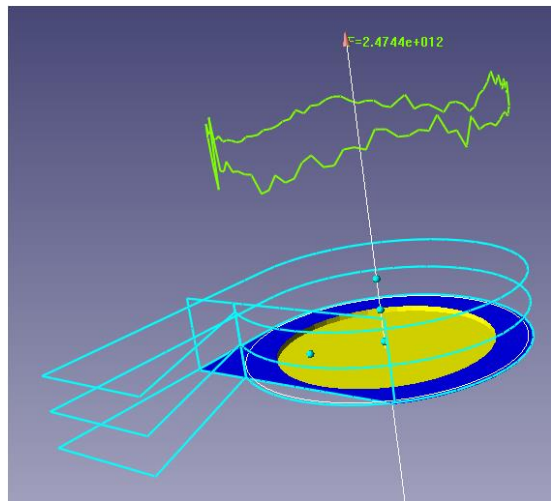

Fig. 11. Structure of the peripheral flow in the vortex chamber (received by the numerical method based on the solution of Navier-Stokes equation system): a - streamlines of the peripheral vortex flow; $b$ - pressure distribution along the housing shell caused by the impact of toroidal vortexes on it

It is possible to provide the operation of toroidal vortex flow by:

- the size of the inlet;

- the direction of inlet installation; 
- eccentricity of the vortex chamber and of the resonator outlet tube;

- (spiral) shell profiling;

- stator system, etc.

\section{Conclusions}

1. It is shown that the sound creation in the vortex chamber takes place at the cost of the interaction between the input and peripheral flows;

2. The parameters of the peripheral flows influence considerably the stability of frequency and of wave amplitude;

3. The formation of two conjugate vortex toroidal flows is due to the following facts:

- deceleration at the beginning of the concave cylindrical section of the deep layers of the flow entering the vortex chamber;

- transformation of the kinetic form of energy into piezometric in the deep layers of the input flow;

- deviation of the upper layers along the parabolic trajectory of the inlet flow from the pressure created by the deep stagnant layers;

- breakthrough of the deep layers of the input stream through two sides of the wall zones;

- combination of flows from the near-wall zones due to the convergence from the mutual ejection effect;

- the movement of the generalized flow along the chord of the vortex chamber until it sticks to the next section of the cylindrical shell;

- subsequent formations of new similar cycles.

4. The inlet potential flow of the concave cylindrical section the of vortex chamber is transformed into a structure consisting of:

- two conjugate helical peripheral flows along the vortex chamber shell;

- circular flow along a parabolic trajectory until alignment with the shell and further along the shell along the circular trajectory;

- passive volumes of liquid from the interaction zone of the circumferential and inlet flows.

5. A computational model of the leakage of a channel flow onto an inclined obstacle, both in the form of a flat and in the form of a cylindrical wall, is proposed, including:

- the condition of bending of the flat flow from the transverse pressure created by the bottom inhibited layers;

- the condition of the minimum energy consumption of the upper layers of the flow around an inclined obstacle: flat - along a tangential trajectory; concave cylindrical - according to the minimum angle of divergence of the flow vector and tangent to the vortex chamber shell.

6. Calculated expressions have been obtained that make it possible to determine the fluid flow rates of each component of the toroidal peripheral flow for different kinematic modes of the inlet flow and different geometry of the vortex chamber.

7. The adequacy is proved by numerical methods based on solving the Navier-Stokes equations in the Flow vision software package.

\section{References}

[1] Potapov U.S. Heat generator and device for heating liquids. Russian patent RU2045715, F25B29/00, 1993.

[2] Agranat B.A., Dubrovin M.N., Khavsky N.N. Fundamentals of physics and technology of ultrasound. Moscow, Russia: Highschool, 1987.

[3] Ivanov Ye.G. The influence of lateral thrust on slot jet of infinite breadth. The improvement of farm machinery performance rating. The proceedings of Federal State Budgetary Educational Institution of Higher Education "Vyatka state agricultural academy". Series 10. Proceeding of the 2 International Research-to-Practice Conference "Science-Technology-Resource-saving, 2009, pp. 29-33. 
[4] Matsuno Y., Fukushima Y., Matsuo Sh., Hashimoto T., Setoguchi T., Kim H.D. Investigation on temperature separation and flow behavior in vortex chamber. Journal of Thermal Science, 24(2), 2015, pp. 149-154.

[5] Matsuo Sh., Matsuno Y., Fukushima Y., Mamun M., Hashimoto T., Setoguchi T., Kim H.D. Experimental Study on Temperature Separation in Vortex Chamber. Procedia Engineering, 105, 2015, pp. 464-471.

[6] Xue Y. The working principle of a Ranque-Hilsch Vortex Tube. School of Mechanical Engineering. The University of Adelaide South. Australia, 2012.

[7] Akhmetov D.G., Akhmetov T.D. Flow structure in a vortex chamber. Journal of Applied Mechanics and Technical Physics, 57(5), 2016, pp. 879-887.

[8] Akhmetov D.G., Akhmetov T.D., Pavlov V.A. Flow Structure in a Ranque-Hilsch Vortex Tube. Doklady Physics, 63(6), 2018, pp. 235-238.

[9] Sudo K., Sumida M., Hibara H. Experimental investigation on turbulent flow in circular-sectioned 90-degree bend. Experiments in Fluids. Vol. 25. Issue 1. 1998, pp. 42-49.

[10] Tanaka M, Ohshima H. Numerical investigation on large scale eddy structure in unsteady pipe elbow flow at high Reynolds number conditions with large eddy simulation approach Journal of Power and Energy Sistems. Vol. 6. Issue 2. 2012, pp. 210-228.

[11] Dutta P., Saha S.K., Nandi N., Pal N. Numerical study on flow separation in 90 pipe bend under high Reynolds number by k-e modeling. Engineering Science and Technology, an International Journal. Vol. 19. Issue 2. 2016, pp. 904-910. 